\title{
Dual coding under conditions of second stimulus modality uncertainty in successive matching tasks
}

\author{
NIGEL HARVEY \\ University College London, London, England
}

\begin{abstract}
Subjects matched successively presented stimuli within and across modalities. In conditions in which they were informed of the modalities of the two stimuli, no differences in matching performance were obtained between the four types of match (visual-visual, auditory-auditory, visual-auditory, and auditory-visual). Thus, there appeared to be no difference between the modalities in ability to perceive or retain the particular stimuli used. In conditions in which subjects were informed of the modality of the first stimulus but only of the modality in which the second stimulus would appear on $80 \%$ of trials, there was again no significant difference between auditory-auditory and visual-visual matching. However, auditory-visual matching was much faster than visual-auditory matching when the second stimulus appeared in the unexpected modality. The results suggest that subjects prepare for both possible types of match when uncertain of the second stimulus modality and that the cross-modal asymmetry reflects the additional attentional load that this incurs.
\end{abstract}

Milewski and laccino (1982) examined intramodal and cross-modal performance asymmetries in a matching task. Subjects matched the lengths of two lines separated by an interstimulus interval (ISI) of 5 or $20 \mathrm{sec}$. Each line was either visually (V) or haptically $(\mathrm{H})$ presented. Some subjects were informed of the modalities of presentation of the two stimuli prior to each trial. Other subjects were not given this information. All subjects showed better VV than $\mathrm{HH}$ matching performance. In contrast, the direction of the cross-modal asymmetry depended on whether or not subjects were informed of stimulus presentation modalities prior to each trial. When they were given this information, HV matching was better than VH matching. When this information was not given, the opposite pattern of results was obtained.

Milewski and Iaccino (1982) argue that the asymmetries in the "informed" condition show that haptic memory is worse than visual memory and that intermodal transfer occurs early when it is required. Thus, information is stored in the modality of the second stimulus $\left(\mathrm{S}_{2}\right)$ during the ISI. This is done even when it means that the inferior haptic memory must be used. Consequently, HV performance is better than VH performance. To account for the reversal of this cross-modal asymmetry when subjects are uncertain of presentation modalities, Milewski and laccino (1982) consider two alternative explanations. I shall

The author's mailing address is: Department of Psychology, University College London, Gower Street, London WCIE 6BT, England. term these the wait-and-see model and the dual-code model.

According to the wait-and-see model, uncertainty concerning modality of $S_{2}$ leads subjects to delay any intermodal transfer of information until $S_{\mathbf{2}}$ arrives. So, in contrast to what happens when subjects are certain of the modality of $S_{2}$, information is stored in the modality of the first stimulus $\left(\mathrm{S}_{1}\right)$ during the ISI. Consequently, worse haptic than visual memory now leads to worse HV than VH performance. (A lack of interaction between ISI and matching condition leads Milewski \& Iaccino, 1982, to regard this worse haptic memory to be more variable or less precise than visual memory, rather than faster decaying.)

According to the dual-code model, uncertainty concerning $S_{2}$ modality leads subjects to maintain $S_{1}$ information in its original modality while simultaneously transferring it into the other modality. As maintenance of haptic information demands more processing capacity than maintenance of the corresponding visual information (Posner, 1967), there is less capacity available for a satisfactory cross-modal transfer in the HV than in the VH condition. Consequently, the trace of $S_{1}$ after transfer is less precise on $\mathrm{HV}$ than on VH trials. So, according to this theory, the intramodal asymmetry is due to a less precise haptic than visual trace (because of haptic memory characteristics), but the cross-modal asymmetry is due to a less precise visual than haptic trace (because haptic storage demands lead to inefficient HV transfer).

Both these accounts can be tested. Consider the wait-and-see model first. If there are neither intra- 
modal nor cross-modal asymmetries when subjects are told the $S_{2}$ presentation modality, then there should be neither of these asymmetries when that information is withheld. Introduction of $S_{2}$ modality uncertainty should not affect basic memory characteristics of the modality-specific stores. Now consider the dual-code model. If neither intramodal nor cross-modal asymmetries are present when subjects are told the $S_{2}$ presentation modality, then no intramodal asymmetry should be present when that information is withheld. However, a cross-modal asymmetry could still appear. This is because even when the memory traces in the two modalities are equally good, maintenance of one of them could take up more processing capacity and hence leave less available for simultaneous intermodal transfer in one direction than in the other. To test these predictions, asymmetries in the conditions in which subjects are informed of $S_{2}$ modality must be minimized. These asymmetries arise from perceptual and memory factors. Perceptual factors can be controlled by adopting a paradigm in which discriminability of stimuli in the two modalities can be varied independently. Memory factors can be controlled by choosing modalities and stimuli for which basic storage characteristics are known to be similar.

With these considerations in mind, Milewski and Iaccino's (1982) paradigm was modified in certain ways:

(1) Audition (A) was substituted for the haptic modality. While haptic memory is known to be poor (Posner, 1967), nonverbal auditory memory is good over short time intervals and should be comparable to nonverbal visual memory. Harris (1952), for instance, found that the difference threshold for pitch increased by only $0.8-3.7 \mathrm{~Hz}$ when ISI was increased from 0 to $15 \mathrm{sec}$. The possibility of effects arising from different memory characteristics was further minimized by fixing ISI at a small value $(500 \mathrm{msec})$ and limiting the set from which stimuli were drawn to two.

(2) In cross-modal conditions, subjects were instructed that a high-pitched tone was equivalent to a high visual position and a low-pitched tone was equivalent to a low visual position and that they were to perform the matching task accordingly. (Pitch of two tones was compared in AA conditions and vertical position of two visual stimuli in VV ones.) Use of this judgment, which is similar to that of matching the pitch of a note to its vertical position on a musical score, allows the experimenter to vary the discriminability of visual and auditory stimuli independently. Here stimuli were chosen so that discriminability was the same in the two modalities. Previous work (Harvey, 1980) has shown that when the particular stimuli employed here were preceded by homomodal warning signals, mean reaction times (RT) required to choose between the two visual ones was 389 msec and mean RT required to choose between the two auditory ones was $384 \mathrm{msec}$.

(3) Milewski and Iaccino's (1982) "uninformed" subjects knew that $S_{2}$ would appear in one modality on $50 \%$ of occasions and in the other modality on the remaining $50 \%$ of occasions. If they are correct in thinking that the results they obtained reflected optional strategies adopted by subjects to deal with uncertainty, then degree of uncertainty may affect strategy adopted and, hence, results obtained. To examine this possibility, degree of uncertainty was varied in the present experiment. In line with Milewski and Iaccino's (1982) procedure, conditions in which subjects were certain of the modalities of both $S_{1}$ and $S_{2}$ were compared with those in which they knew the modality of $S_{1}$ but were uncertain of that of $S_{2}$. However, these latter conditions differed from those in Milewski and Iaccino's (1982) experiment. Subjects were told that $S_{2}$ would appear in one modality (the "expected" modality) on $80 \%$ of occasions and in the other modality (the "unexpected" modality) on the remaining $20 \%$ of occasions.

\section{METHOD}

\section{Subjects}

Three male and five female subjects, aged between 19 and 25 years, served for two practice and two experimental sessions of 70 min duration each. Each subject was paid a total of $\boldsymbol{2 1 . 5 0}$. All subjects were right-handed.

\section{Apparatus}

Auditory stimuli were generated by Advance Instruments J2-E oscillators and delivered to Sony DR-3C stereo headphones via a multichannel gate unit. Visual stimuli were displayed on a Tektronix RM 561A oscilloscope with a P15 phosphor T5032-31-1 cathode ray tube. A subject sat alone in a dim, sound-dampened cubicle and responded by pressing piano-type keys on a bench in front of him or her. Control of the auditory gate unit and of the oscilloscope and recording and timing of responses were all performed on-line by an LINC-8 computer.

\section{Stimuli}

The high and low auditory stimuli were pure sinusoids with frequencies of 1100 and $900 \mathrm{~Hz}$, respectively. Both signals were binaural, gated at random phase and presented at a loudness level of $70 \mathrm{~dB}$ SPL. The high and low visual stimuli were squares presented an equal distance above and below the midpoint of the central vertical axis of the oscilloscope screen. The visual angle subtended by each square was $35 \mathrm{~min}$ of arc, and the visual angle subtended by the distance between their midpoints was $7^{\circ} 30^{\prime}$.

\section{Experimental Design}

Each trial consisted of an $S_{1}$ lasting $500 \mathrm{msec}$, followed first by an ISI of 500 msec and then by an $S_{2}$ lasting $500 \mathrm{msec}$. After that sequence of events, the subject was given $2 \mathrm{sec}$ in which to respond by pressing the appropriate key. Within each block of $\mathbf{4 0}$ trials, the four combinations of $S_{1}$ and $S_{2}$ categories (high-high, low-low, high-low, and low-high) were presented randomly, with the constraint that they appeared an equal number of times. $S_{2}$ modality was constant within any particular block of trials. In the four certain conditions (VV, AA, AV, VA), $S_{2}$ modality was also constant within a trial block. However, within each block of the four uncertain conditions $\left[V\left(V_{10} / A_{20}\right), A\left(A_{80} / V_{20}\right), A\left(V_{10} / A_{20}\right)\right.$, and $\left.V\left(A_{60} / V_{20}\right)\right], S_{1}$ was either visual on a randomly selected $80 \%$ of 
trials and auditory on the rest or auditory on a randomly selected $80 \%$ of trials and visual on the rest. Thus, different levels of $S_{2}$ modality uncertainty were represented by different prior probabilities that $S_{2}$ would be in the modality of actual presentation. This probability $\left[P\left(M_{2}\right)\right]$ took the value 1.0 in the four certain conditions and the values 0.8 and 0.2 for the expected and unexpected modality, respectively, of the four uncertain conditions. Within each of the four sessions, two blocks of each of these eight conditions were presented. Order of conditions was balanced across subjects.

\section{Procedure}

After entering the experimental cubicle, the subject was shown examples of the stimuli to be used. He (or she) was told that on each trial he was to match the two stimuli by pressing the key under his right forefinger if they were both high or both low and the key under his left forefinger if one was high and the other was low. He was asked to respond as fast as possible while trying to keep his errors below about two per block of 40 trials.

After each of the first two sessions, which were considered solely as practice, each subject was given feedback regarding his RTs and error rates. In the second two sessions, $\mathbf{1 0}$ trials of practice were given on each condition prior to its commencement. The four sessions were given on separate days, but in no case did more than 2 days intervene between one session and the next. Only data from the last two sessions are analyzed below.

\section{RESULTS}

\section{Reaction Times}

Mean RTs were subjected to a five-way analysis of variance, with subjects as a random effects factor and $S_{1}$ modality, intramodal vs. cross-modal matching, same vs. different, and $P\left(M_{2}\right)$ as fixed effects factors. $\mathbf{P}\left(\mathbf{M}_{2}\right)$ had a highly significant effect on $R T$ $[F(2,14)=100.11, p<.0001]$. The appearance of cross-modal asymmetries as this variable decreased produced interactions between it and both $S_{1}$ modality $[F(2,14)=15.47, p<.0005]$ and intramodal vs. cross-modal matching $[\mathrm{F}(2,14)=5.03, \mathrm{p}<.05]$ and between all three of these variables $[F(2,14)=$ $5.40, p<.05$ ]. Newman-Keuls test failed to reveal any significant differences between the four types of matching when $P\left(M_{2}\right)$ was 1.0 or 0.8 . However, when $\mathrm{P}\left(\mathrm{M}_{2}\right)$ was 0.2 , AV matching was found to be significantly faster than VA and AA matching at the $1 \%$ level and significantly faster than VV matching at the $5 \%$ level. When $P\left(M_{2}\right)$ was 0.2 , all four types of matching were significantly slower at the $1 \%$ level than they were when $P\left(M_{2}\right)$ was 0.8 . When $P\left(M_{2}\right)$ was
$0.8, A A, V A$, and AV matching were all significantly slower than they were when $\mathbf{P}\left(\mathbf{M}_{\mathbf{2}}\right)$ was $\mathbf{1 . 0}$.

There was some evidence that intramodal same RT (419 msec) was slower than intramodal different RT (416 msec) but that cross-modal same RT (421 msec) was faster than cross-modal different RT $(436 \mathrm{msec})$ $[F(1,7)=8.55, p<.025]$. That the effect is related to the expected rather than the actual relation between the modalities of two stimuli is shown by its reversal when $P\left(M_{2}\right)=0.2[F(2.14)=4.34, p<.05]$. As $P\left(M_{2}\right)$ decreased from 1.0 through 0.8 to 0.2 , the (same RT - different RT) difference decreased from $11 \mathrm{msec}$ through $9 \mathrm{msec}$ to $-12 \mathrm{msec}$ for intramodal trials but increased from $-24 \mathrm{msec}$ through $-27 \mathrm{msec}$ to $5 \mathrm{msec}$ for cross-modal ones. As there was no evidence that same vs. different interacted with the performance asymmetries reported in the previous paragraph and of prime interest here, data were collapsed over this variable for presentation in Table 1.

\section{Error Rates}

After an arcsin transformation, error rates were subjected to an analysis of variance with the same factors as those employed for the RT analysis. The effect of $P\left(M_{2}\right)$ reached significance $[F(2,14)=6.36$, $\mathrm{p}<.025]$. Error rates were lower for "same" $(2.4 \%)$ than for "different" $(4.6 \%)$ responses $[F(1.7)=5.62$, $\mathrm{p}<.05]$ and this difference was greater on crossmodal $(3.0 \%)$ than on intramodal $(1.4 \%)$ trials $[F(1,7)=7.41, p<.05]$.

\section{DISCUSSION}

Table 1 and the above analysis reveal that when subjects were informed of the modality of $S_{2}$, mean intramodal matching RTs were within a few milliseconds of one another and corresponding error rates were likewise virtually identical. Cross-modal matching RTs were even closer together and error rates were again very similar. In contrast, asymmetries appeared in the data when subjects were uncertain of the $\mathbf{S}_{\mathbf{2}}$ modality. Intramodal $\mathrm{RT}$ asymmetries were quite small and probably counterbalanced slight asymmetries in the error rates in the opposite direction. The cross-modal matching asymmetry was particularly evident when $S_{2}$ appeared in the "unex-

Table 1

Mean RTs (in Milliseconds) and Error Rates (in Percentages) for Each of the Four Modality Combinations and for Each of the Three Levels of the Prior Probability of $\mathrm{S}_{2}$ Being in Modality of Actual Presentation

\begin{tabular}{|c|c|c|c|c|c|c|}
\hline \multirow[b]{3}{*}{ Matching Condition } & \multicolumn{6}{|c|}{ Prior Probability of $S_{2}$ Being in Modality of Actual Presentation } \\
\hline & \multicolumn{2}{|c|}{1.0} & \multicolumn{2}{|c|}{0.8} & \multicolumn{2}{|c|}{0.2} \\
\hline & RT & Error & RT & Error & RT & Error \\
\hline Visual-Visual & 339 & 3.3 & 385 & 2.9 & 510 & 3.5 \\
\hline Auditory-Auditory & 333 & 3.5 & 415 & 1.9 & 529 & 2.0 \\
\hline Visual-Auditory & 361 & 3.7 & 419 & 3.4 & 546 & 5.1 \\
\hline Auditory-Visual & 360 & 3.1 & 403 & 3.1 & 474 & 5.5 \\
\hline
\end{tabular}


pected" modality. AV matching RT was then $72 \mathrm{msec}$ faster than VA matching RT.

Results such as these are difficult to reconcile with the wait-and-see model. By far the most parsimonious explanation for the lack of intramodal and cross-modal asymmetries when subjects were informed of the $S_{2}$ modality is that perception and memory of the stimuli in the two modalities were equally good. Perception produced traces of visual and auditory stimuli with equal precision and, if these traces decayed during the ISI, then they did so at the same rate. The wait-and-see model requires an absence of such equalities in order to account for the asymmetries observed under $S_{\mathbf{2}}$ modality uncertainty. Consequently, it cannot explain the cross-modal asymmetry obtained here when $S_{2}$ appeared in the unexpected modality. An attentional explanation, of which Milewski and Iaccino's (1982) dual-coding model is an example, is more likely to provide a satisfactory account of the present data.

Two types of performance decrement can be extracted from Table 1 for each of the four types of match (VV, AA, VA, AV). The decrement associated with the appearance of $S_{2}$ in the "expected" modality of $S_{2}$ uncertain conditions $\left(D_{E}\right)$ is obtained by subtracting RT for a particular type of match when $P\left(M_{2}\right)=1.0$ from RT for that same type of match when $\mathbf{P}\left(\mathbf{M}_{2}\right)=$ 0.8 . The decrement associated with the appearance of $S_{2}$ in the "unexpected" modality of $S_{2}$ uncertain conditions $\left(D_{U}\right)$ is obtained by subtracting $R T$ for a particular type of match when $P\left(M_{2}\right)=1.0$ from RT for that same type of match when $\mathbf{P}\left(\mathbf{M}_{2}\right)=0.2$. Each of the four $S_{2}$ modality uncertain conditions $\left[V\left(V_{B 0}\right.\right.$ ] $\left.\left.A_{20}\right), A\left(A_{80} / V_{20}\right), V\left(A_{80} / V_{20}\right), A\left(V_{80} / A_{20}\right)\right]$ yields a $D_{E}$ associated with one type of match and a $D_{U}$ associated with a different one. Thus, for example, the $\mathrm{V}\left(\mathrm{V}_{\mathrm{so}} / \mathrm{A}_{20}\right)$ condition gives a $D_{\mathrm{E}}$ for $\mathrm{VV}$ matches of $46 \mathrm{msec}$ and a $D_{U}$ for VA matches of $185 \mathrm{msec}$. When the $D_{E}$ and $D_{U}$ obtained from the same condition are plotted against one another for each of the four $S_{2}$ modality uncertain conditions, a strong inverse relation becomes evident (Figure 1). The correlation $(\mathrm{r}=-0.992)$ is highly significant $[\mathrm{t}(2)=11.407$, $\mathrm{p}<.01]$.

Figure 1 represents an RT exchange function of the type examined by Audley, Caudrey, Howell, and Powell (1975), and its linear nature is consistent with the results they obtained for stimuli distinguished by their position in a spatial array.

The ordering of decrements depicted in Figure 1 cannot be explained merely by assuming that attention is directed towards vision when subjects are uncertain of $\mathbf{S}_{\mathbf{2}}$ modality. Such a supposition would account for why $D_{E}$ is less in $A\left(V_{80} / A_{20}\right)$ and $V\left(V_{s 0} /\right.$ $\left.A_{20}\right)$ conditions than in $A\left(A_{100} / V_{20}\right)$ and $V\left(A_{80} / V_{20}\right)$ conditions, and also why $D_{U}$ is more in the former pair of conditions than in the latter pair. However, it would not explain why $D_{E}$ is so very much larger and $D_{U}$ is

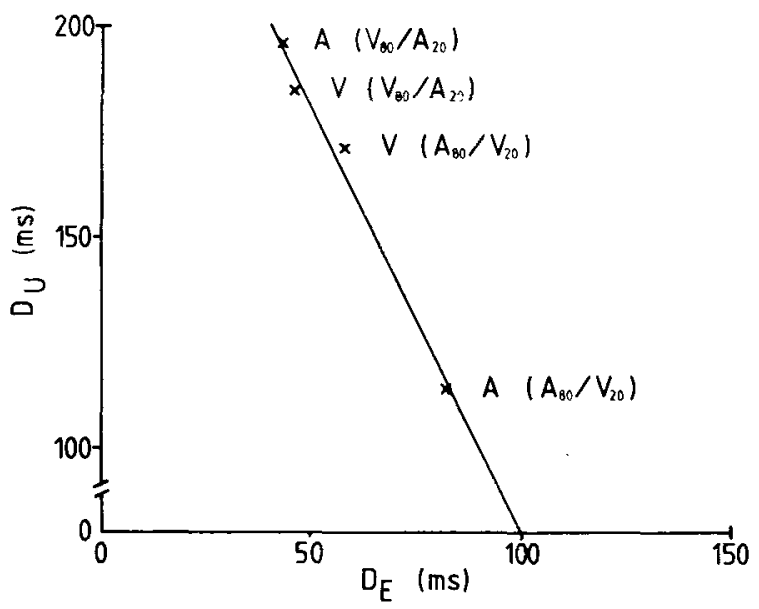

Figure 1. Graph of mean RT decrement obtained when $S_{2}$ appeared in the "unexpected" modality $\left(D_{Y}\right)$ against mean $R T$ decrement obtained when $S_{2}$ appeared in the "expected" modality $\left(D_{\mathrm{E}}\right)$ for each of the four $S_{2}$ modality uncertain conditions [ $V\left(V_{\mathbf{w 0}_{0}} / \boldsymbol{A}_{\mathbf{2 0}}\right)$, $\left.A\left(A_{10} / V_{20}\right), V\left(A_{20} / V_{20}\right), A\left(V_{10} / A_{20}\right)\right]$. Decrements are relative to performance for the same type of match (i.e., VV, AA, VA, or AV) when subjects were certin of the $S_{2}$ modality.

so very much smaller in the $A\left(A_{80} / V_{20}\right)$ than in the $V\left(A_{80} / V_{20}\right)$ condition or why differences in $D_{E}$ and $D_{U}$ are also evident when conditions $A\left(V_{20} / A_{20}\right)$ and $\mathrm{V}\left(\mathrm{V}_{\mathrm{BO}} / \mathrm{A}_{\mathbf{2 0}}\right)$ are compared. $\left(\mathrm{D}_{\mathrm{E}}\right.$ and $\mathrm{D}_{\mathrm{U}}$ are decrement measures and, hence, take into account the basic cost of having to match cross-modally rather than intramodally.) In fact, it appears that the identical ordering of $D_{E}$ and $D_{U}(A A>V A>V V>A V)$ is consistent with a dual code model in which fixed proportions of attention are devoted to preparing for stimuli in the "expected" and "unexpected" modalities and in which the attention demands for the four types of match are ordered as above.

Norman and Bobrow (1975) have termed performance data-limited when supply of attention or resources exceeds demands, and resource-limited when it does not. Performance in $S_{\mathbf{2}}$ modality-certain conditions was data-limited, and so the differing attentional demands of the four types of match did not affect the data. Attentional sharing necessitated by dual coding resulted in resource-limited performance in $S_{2}$ modality-uncertain conditions. The attention devoted to preparing for each of the two possible types of match reflected their relative likelihoods of being required. As performance was resourcelimited, it was worse for the less likely match. Thus, values of $D_{U}$ are greater than corresponding values of $D_{E}$. The slope of the curve in Figure 1 would have been less had the likelihood ratio of $S_{2}$ appearing in the two modalities been closer to unity.

Suppose that the proportions of attention devoted to preparing for matching stimuli in the "expected" and "unexpected" modalities were constant across all four $S_{2}$ modality-uncertain conditions. For ex- 
ample, $80 \%$ of available resources may have been dedicated to preparing for a match with a stimulus in the "expected" modality and $20 \%$ to preparing for a match with a stimulus in the "unexpected" modality. As performance is resource-limited, level achieved for a particular type of match corresponds, in some way, to the proportion of its resource demands that are satisfied by the supply. Maintaining a trace in auditory memory and monitoring audition for an AA match is much more attention-demanding than transferring information to vision, maintaining it there, and monitoring that modality for an AV match. Thus, with some fixed supply of attention (either $80 \%$ or $20 \%$ of total resources), a greater proportion of AV match demands are satisfied than are AA match demands. Hence, AV matches are performed much better than AA matches for a given level of $P\left(M_{2}\right) . D_{E}$ and $D_{U}$ for $A A$ matches are greater than $D_{E}$ and $D_{U}$ for $A V$ matches by 39 and $82 \mathrm{msec}$, respectively. Similarly, maintaining a trace in visual memory and monitoring vision for a VV match was slightly less attention demanding than transferring information to audition, maintaining it there, and monitoring that modality for a VA match. Thus, with some fixed amount of attention (either $80 \%$ or $20 \%$ of total resources), a greater proportion of VV than VA demands on attention are satisfied by the supply. Hence, VV matches were performed slightly better than VA matches for a given level of $P\left(M_{2}\right)$. $D_{E}$ and $D_{U}$ for $V V$ matches are less than $D_{E}$ and $D_{U}$ for VA matches by 12 and $16 \mathrm{msec}$, respectively.

Thus, the present data support the view that datalimited maintenance of an auditory trace is as good as data-limited maintenance of a visual one. However, when performance is resource-limited and resources are fixed at some level, the higher resource demands of auditory than visual trace maintenance result in less precise auditory than visual traces being present at the end of the ISI. The greater difference between AA and AV performance decrements than between VV and VA performance decrements may be related to this. If AV transfer was carried out early in the ISI to gain maximum benefit from the superiority of visual memory under resourcelimited conditions, then the difference between AA and $A V$ performance caused by memory factors would have been maximized. If the VA transfer was carried out late in the ISI for the same reason, then the performance difference between VV and VA matching would have been minimized. This suggestion could be tested by repeating the present experiment but including ISI as an independent variable. The greater difference between AA and AV decrements than between VV and VA decrements should become accentuated with increase in ISI.

Finally, brief mention should be made of the subsidiary finding that performance on "different" trials deteriorated relative to that of "same" trials when subjects were required to match cross-modally rather than intramodally. This effect, apparently independent of the performance asymmetries discussed above, replicates one reported by Harvey (1973). Its reversal when $\mathbf{P}\left(\mathbf{M}_{2}\right)=0.2$ indicates that it arises from the expected, rather than from the actual, relation between the modalities of the two stimuli. An ad hoc explanation of it, based on recent accounts of the Stroop effect (Dyer, 1973; Glaser \& Dolt, 1977) and other phenomena demonstrating the interfering effects of irrelevant information (Harvey, 1980), is as follows. Subjects automatically categorize the modalities of the two stimuli as "same" on intramodal trials and as "different" on cross-modal ones. To ensure that they do not respond on the basis of this automatic, but irrelevant, comparison, responses associated with the "same" category are delayed on intramodal trials and those associated with the "different" category are delayed on cross-modal ones. This delay allows subjects to check that the response is being made on the basis of the relevant betweenposition match rather than on the basis of the irrelevant between-modality one. To be effective, the delay is instigated prior to the automatic analysis of the modality of $S_{2}$ and on the basis of the expected relation between the modalities of the two stimuli. The delay itself produced the observed RT effects and the fact that it was occasionally insufficient for checking to be accomplished produced the corresponding error effects.

\section{RETERENCES}

Audley, R. J., Caudney, D. J., Howell, P., \& Powell, D.J. Reaction time exchange functions in matching tasks. In P.M.A. Rabbitt \& S. Dornic (Eds.), Attention and performance V. London: Academic Press, 1975.

DYER, F. N. The Stroop phenomenon and its use in the study of perceptual, cognitive and response processes. Memory \& Cognition, 1973, 1, 106-120.

Glaser, W. R., \& Dout, M. O. A functional model to localize the conflict underlying the Stroop phenomenon. Psychological Research, 1977, 39, $287-316$.

HARRIs, J. D. The decline in pitch discrimination with time. Journal of Experimental Psychology, 1952, 43, 96-99.

Harvey, N. Does intermodal equivalence exist between heteromodal stimulus dimensions or between stimulus values on those dimensions?Quarterly Journal of Experimental Psychology, 1973, 25, 476-491.

HARveY, N. Non-informative effects of stimuli functioning as cues. Quarterly Journal of Experimental Psychology, 1980, 32, 413-425.

MILEWSK1, A. E., \& IAccrNo, J. Strategies in cross-modality matching. Perception \& Psychophysics, 1982, 31, 273-275.

Norman, P. A., \& Bobrow, D. G. On data-limited and resourcelimited processes. Cognitive Psychology, 1975, 7, 44-64.

Posner, M. Characteristics of visual and kinaesthetic memory codes. Journal of Experimental Psychology, 1967, 75, 103-107.

(Manuscript received July 23, 1982; revision accepted for publication March 23, 1983.) 